\title{
Differential effect of high dietary fat intakes on haemorheological parameters in rats
}

\author{
Cheng-Jeng Tai ${ }^{1}$, Chao-Hsiang Chen ${ }^{2,3}$, Hui-Hsin Chen ${ }^{4}$ and Hong-Jen Liang ${ }^{4} *$ \\ ${ }^{1}$ Section of Hematology-Oncology, Department of Medicine, Taipei Medical University Hospital, Taipei, Taiwan, ROC \\ ${ }^{2}$ Department of Food Science and Biotechnology, National Chung Hsing University, Taichung 40227, Taiwan, ROC \\ ${ }^{3}$ Ko Da Pharmaceutical Co. Ltd, Ping-Cheng, Taoyuan, Taiwan, ROC \\ ${ }^{4}$ Department of Food Science, Yuanpei University, No. 306, Yuanpei Street, Hsinchu, Taiwan, ROC \\ (Received 28 May 2009 - Revised 6 October 2009 - Accepted 7 October 2009 - First published online 30 November 2009)
}

High dietary intake of fats has been thought to be one of the major risk factors for the development of CVD. Less is known about the possible influence of fats from various sources on haemorheological abnormalities, which are considered an important factor in the pathogenesis of these diseases. The goal of the present study was to investigate effects of high-fat diets enriched in unsaturated fatty acids (USFA), SFA or trans-fatty acids (TFA), respectively, on haemorheological parameters in rats. Wistar female rats were divided into four groups and fed diets based on the AIN-93M formulation containing approximately $10 \%$ energy from soyabean oil (control group) or $40 \%$ energy from soyabean oil (USFA), palm oil (SFA) and vegetable shortening (TFA) for 8 weeks. The results showed that rats fed high-fat diets exhibited significant increases in serum TAG levels $(P<0 \cdot 01)$, plasma viscosity $(P<0 \cdot 01)$, whole blood viscosity $(P<0.01)$ and internal viscosity $(P<0 \cdot 01)$ compared to the controls. The TFA group showed a significant decrease in erythrocyte deformability $(P<0 \cdot 01)$ and increase in internal viscosity $(P<0 \cdot 01)$ compared with the other groups. In addition, a significant increase in blood levels of free radicals $(P<0 \cdot 01)$ was found in the TFA group, suggesting that the attack of oxygen-free radicals could be responsible for the impaired erythrocyte deformability. These impairments could be partly responsible for the development of various circulatory disorders. The present haemorheological study provides additional insights into the potential adverse effects of trans-fat and high-fat diets on haemorheological parameters.

High fat intake: Trans-fatty acids: SFA: Unsaturated fatty acids: Blood viscosity: Plasma viscosity: Erythrocyte deformability: Erythrocyte aggregation: Free radicals

Intake of high levels of dietary fat is closely linked with the pathogenesis of $\mathrm{CVD}^{(1,2)}$. While most studies of these diseases have focused on biochemical indices or those related to the vascular endothelium, haemorheological abnormalities may underlie the pathophysiologic process be predictors of risk of $\mathrm{CHD}^{(3-5)}$. A number of studies have shown an association between a high intake of dietary fats and haemorheological abnormalities in both animals and human subjects, although the specific sources of fats were not identified. In general, high intake of dietary fats markedly impairs haemorheological properties causing, for example, reduced fluidity of whole blood $^{(6)}$, increases in LDL leading to increased erythrocyte aggregation, and, thus, increased blood viscosity and decreased blood flow ${ }^{(7)}$. In addition, haemorheological abnormalities including elevated viscosity of blood and plasma, reduced erythrocytes deformability and intensified erythrocyte aggregation have also been found in obese individuals with hyperlipidaemia ${ }^{(8,9)}$. Elevated blood and plasma viscosity as well as impaired erythrocyte deformability might produce retardation and stoppage of the flow of erythrocytes in microvessels.
Intensified erythrocyte aggregation resulting in subsequent local accumulation has been shown to be a key factor disturbing the microcirculation ${ }^{(3-5)}$. These cumulative effects of haemorheological abnormalities appear in the form of a disturbed blood flow profile resulting in the development or aggravation of various circulatory disorders such as hypertension, thrombosis, myocardial infarction and cerebrovascular diseases.

High dietary fat intake is generally associated with increased consumption of industrially produced foods and the accompanying large amounts of partially hydrogenated vegetable oils and fats, especially trans-fats ${ }^{(10)}$. Dietary trans-fatty acids (TFA) are primarily formed during the process of partial hydrogenation, in which liquid vegetable oils are converted to solid fats to give many foods important functional and physical characteristics that affect texture and sensory properties as well as stability against rancidity. Accumulating evidence has consistently shown the existence of a significant relationship between the intake of dietary TFA and the risk of cardiovascular disorders $^{(10)}$ through pathways such as increased lipoprotein ${ }^{(11)}$ and blood TAG concentrations ${ }^{(12)}$, systemic inflammation ${ }^{(13)}$

Abbreviations: Con, control group; DI, deformability index; Hct, haematocrit; HDL-C, HDL cholesterol; LDL-C, LDL cholesterol; MCH, mean cell Hb; MCHC, $\mathrm{MCH}$ concentration; $T_{K}$, internal viscosity of erythrocytes (or erythrocyte rigidity); TC, total cholesterol; TFA, trans-fatty acids; USFA, unsaturated fatty acids.

* Corresponding author: Dr Hong-Jen Liang, fax +886 3 6102342, email jenliang@mail.ypu.edu.tw 
and endothelial cell dysfunction ${ }^{(14,15)}$. However, relatively little information is available on the underlying mechanisms of action of dietary TFA on haemorheological properties, which are also correlated with various cardiovascular events. Additionally, clear results from comparisons of the effects of diets comprising different lipids, such as dietary fats enriched in unsaturated fatty acids (USFA), SFA or TFA, haemorheological properties are lacking.

The purpose of the present study was to investigate whether various oils enriched in USFA, SFA or TFA had differential effects on the haemorheological parameters of rats fed high-fat diets in relation to their effects on blood biochemical and haematological properties. In addition, there is a strong suggestion that oxidative stress is a key factor leading to abnormal haemorheological parameters ${ }^{(16,17)}$. However, data on the effects of oxidative stress in relation to a high-fat intake on haemorheological abnormalities are still needed. Therefore, the content of oxygen-free radicals in the blood of rats fed with high-fat diets was measured to understand whether oxidative stress was related to the effect of high dietary fat intake on haemorheological properties.

\section{Materials and methods}

\section{Animals and treatments}

The protocol of the present study was approved by the Yuanpei University Animal Care and Ethics Committee, and all the animal studies were conducted following the recommendations provided by the European Convention for the protection of Vertebrate Animals used for Experimental and Scientific purposes (Council of Europe no. 123, Strasbourg, 1985). Female Wistar rats at 8 weeks of age were supplied by the Animal Laboratory Service of the Taipei Medical University. They were housed in animal quarters under controlled conditions $\left(22-25^{\circ} \mathrm{C}\right.$ and $12 \mathrm{~h}$ light-dark cycle) in individual cages. Animals were allowed to acclimatise to their new conditions for a week before the study. Then the animals were randomly divided into four groups of ten, matched by mean weight. Rats were fed for 8 weeks until sacrifice.

All diets were nutritionally adequate based on the AIN-93M and differed only in the amount and types of fats. The detailed compositions of these diets are described in Table 1. The control (Con) diet contained $40 \mathrm{~g}$ fat $/ \mathrm{kg}$, which accounted for $10 \%$ of the total energy $(15072.85 \mathrm{~kJ} / \mathrm{kg}(3600 \mathrm{kcal} / \mathrm{kg}))$; and the other three isoenergetic experimental diets were enriched by replacing carbohydrate with fats of different compositions to attain $210 \mathrm{~g} / \mathrm{kg}$ (approximately $40 \%$ energy as fat), which exceeded the essential fatty acid recommendations. The fat sources in three experimental diets, USFA, SFA and TFA, were soyabean oil, palm oil and vegetable shortening, respectively. The fatty acid composition of the experimental fats was determined by standard gas chromatographic separation of fatty acid methyl esters. The fatty acid composition of the three experimental fats is

Table 1. Nutrient composition of the experimental diets in the control group (Con) and the experimental groups (unsaturated fatty acids (USFA), SFA and trans-fatty acids (TFA)) $)^{*}$

\begin{tabular}{|c|c|c|c|c|}
\hline & \multicolumn{4}{|c|}{ Diet } \\
\hline & Con & USFA & SFA & TFA \\
\hline Energy (kJ/kg) & $15072 \cdot 85$ & $15072 \cdot 85$ & $15072 \cdot 85$ & $15072 \cdot 85$ \\
\hline \multicolumn{5}{|l|}{ Nutrient } \\
\hline Maize starch (g/kg diet) & 465.69 & 295.69 & 295.69 & 295.69 \\
\hline Dextrin ( $\mathrm{g} / \mathrm{kg}$ diet) & 150 & 150 & 150 & 150 \\
\hline Casein (g/kg diet) & 140 & 140 & 140 & 140 \\
\hline Sucrose (g/kg diet) & 100 & 100 & 100 & 100 \\
\hline Cellulose (g/kg diet) & 50 & 50 & 50 & 50 \\
\hline$t$-Butylhydroquinone-BHT & 0.008 & 0.008 & 0.008 & 0.008 \\
\hline Mineral mixture (g/kg diet) & 35 & 35 & 35 & 35 \\
\hline Vitamin mixtures (g/kg diet) & 10 & 10 & 10 & 10 \\
\hline L-Cys (g/kg diet) & $1 \cdot 8$ & $1 \cdot 8$ & $1 \cdot 8$ & $1 \cdot 8$ \\
\hline Choline bitatrate ( $\mathrm{g} / \mathrm{kg}$ diet) & 2.5 & $2 \cdot 5$ & $2 \cdot 5$ & 2.5 \\
\hline Experimental fats used in the animal diets ( $\mathrm{g} / \mathrm{kg}$ diet) & $\begin{array}{c}\text { Soyabean oil } \\
40\end{array}$ & $\begin{array}{l}\text { Soyabean oil } \\
210\end{array}$ & $\begin{array}{l}\text { Palm oil } \\
210\end{array}$ & $\begin{array}{l}\text { Vegetable shortening } \\
210\end{array}$ \\
\hline \multicolumn{5}{|l|}{ Fatty acids in the experimental fats (\%) } \\
\hline $16: 0$ & \multicolumn{2}{|c|}{$11 \cdot 15$} & $38 \cdot 75$ & $14 \cdot 43$ \\
\hline $16: 1$ & \multicolumn{2}{|c|}{ ND } & 0.12 & ND \\
\hline $18: 0$ & \multicolumn{2}{|c|}{$4 \cdot 24$} & $4 \cdot 12$ & $12 \cdot 39$ \\
\hline $18: 1 t$ & \multicolumn{2}{|c|}{ ND } & ND & $11 \cdot 3$ \\
\hline $18: 1 c$ & \multicolumn{2}{|c|}{24.01} & $42 \cdot 86$ & $38 \cdot 01$ \\
\hline $18: 2$ & \multicolumn{2}{|c|}{52.98} & $12 \cdot 11$ & $31 \cdot 22$ \\
\hline $18: 3$ & \multicolumn{2}{|c|}{$5 \cdot 78$} & 0.18 & 2.65 \\
\hline $20: 0$ & \multicolumn{2}{|c|}{ ND } & ND & ND \\
\hline $20: 1 t$ & \multicolumn{2}{|c|}{ ND } & ND & ND \\
\hline $20: 1 c$ & \multicolumn{2}{|c|}{0.69} & 0.24 & 0.14 \\
\hline $22: 0$ & \multicolumn{2}{|c|}{0.48} & ND & 0.38 \\
\hline $24: 0$ & \multicolumn{2}{|c|}{0.16} & ND & 0.12 \\
\hline$\%$ TFA & \multicolumn{2}{|c|}{ ND } & ND & $11 \cdot 3$ \\
\hline$\%$ SFA & \multicolumn{2}{|c|}{$16 \cdot 7$} & $44 \cdot 3$ & $28 \cdot 0$ \\
\hline
\end{tabular}

BHT, butylated hydroxytoluene, ND, not determined; $c$, cis-double bound; $t$, trans-double bound

${ }^{\star}$ Results are expressed as the mean proportions from three replicates. 
summarised in Table 1 . The diets were freshly prepared every $3 \mathrm{~d}$ during the experimental period using the same batch of raw materials and stored at $4^{\circ} \mathrm{C}$. The animals had free access to water and food. The food intake of each individual rat was recorded daily. Body weight of the animals was recorded weekly.

\section{Blood biochemical measurements}

After an overnight fast $(8 \mathrm{~h})$, the rats were anaesthetised with chloral hydrate $(250 \mathrm{mg} / \mathrm{kg}$, intraperitoneally), and blood was obtained via cardiac puncture. Fresh blood samples from the rats were collected into plastic test-tubes containing EDTA $(1.5 \mathrm{mg} / \mathrm{ml})$ as an anticoagulant. Lipid levels including total cholesterol (TC), which comprised chylomicrons, VLDL, LDL and HDL, and HDL cholesterol (HDL-C) were analysed in plasma samples by enzymatic-colorimetric methods with a Hitachi 7450 analyzer, and TAG levels were measured by a Roche Modular P800 automatic analyzer (Roche Diagnostics, Meylan, France). The concentration of LDL cholesterol (LDL-C) was estimated with the Friedewald formula:

$$
\mathrm{LDL}-\mathrm{C}=\mathrm{TC}-(\mathrm{TAG} / 5+\mathrm{HDL}-\mathrm{C}),
$$

where TAG values $<4000 \mathrm{mg} / \mathrm{l}(<400 \mathrm{mg} / \mathrm{dl})$.

\section{Haematological measurements}

Blood cell counts and other haematological data such as haematocrit $(\mathrm{Hct})$, mean cell volume, mean cell $\mathrm{Hb}(\mathrm{MCH})$ and $\mathrm{MCH}$ concentration (MCHC) were determined by a Scil Vet ABC automatic cell counter (Scil Animal Care Company, Holtzheim, France). Plasma was separated from blood by centrifugation at $1500 \mathrm{~g}$ for $10 \mathrm{~min}$. Fibrinogen levels were measured by a Sysmex CA-500 (Diamond Diagnostics, Hollingston, MA, USA) using reagents from Dade Behring, Inc. (Deerfield, IL, USA), in accordance with the manufacturer's instructions.

\section{Plasma and blood viscosity}

Plasma and blood viscosity were measured using a Rheostress 1 double cone viscometer (HAAKE Mess-Technik, Karlsruhe, Germany) with a cone angle of $1^{\circ}$ at $310 \mathrm{~K}$. The serial blood viscosities at different shear rates were determined via a testing programme. Shear rates of 600,300 and $35 \mathrm{~s}^{-1}$ reflecting high, medium and low shear rates were estimated ${ }^{(18)}$. Erythrocyte rigidity $\left(T_{\mathrm{K}}\right)$ at a shear rate of 600 and $300 \mathrm{~s}^{-1}$ was calculated according to the equation of Dintenfass ${ }^{(19)}$ :

$$
T_{\mathrm{K}}=\frac{\eta r^{0 \cdot 4}-1}{\eta r^{0 \cdot 4} \times \mathrm{Hct}},
$$

where $\eta r$ was the relative blood viscosity $\left(\eta_{600}\right.$ blood or $\eta_{300}$ $\mathrm{blood} / \eta$ plasma).

\section{Deformability and aggregation of erythrocytes}

The measurement of erythrocyte deformability was based on a laser diffraction method in which the laser beam traverses the diluted blood suspension $(0.8 \mathrm{ml})$, and the ability of an erythrocyte to deform in a shear field is monitored by the laser-generated diffraction patterns of the erythrocytes. The diffraction pattern is projected on a screen monitored by photoelectric sensors linked to a frame grabber integrated in the computer. The best-fitting ellipse is determined, and this represents the deformed erythrocyte. The long and short axes, $A$ and $B$, respectively, of this ellipse are then used to calculate the deformability index (DI) as a function of applied shear stress $(30 \mathrm{~Pa})$ :

$$
\mathrm{DI}=\frac{A-B}{A+B} .
$$

Before the measurement, the erythrocytes were suspended in a PBS solution with $5.5 \%$ polyvinylpyrrolidone at the optimal Hct. More detailed information regarding the method has been described elsewhere ${ }^{(20)}$. For the erythrocyte aggregation test, $0.8 \mathrm{ml}$ of an EDTA blood sample was placed into the plate where the diffraction of the laser radiation of the undiluted blood samples was used to estimate the degree of aggregation. The signal obtained from the intensity of the back-scattered light measured by the photoelectric sensors was further processed by the computer to estimate the degree of aggregation. In the present model, the optional disaggregation shear rate and its duration before stopping the motor were generally set at $600 \mathrm{~s}^{-1}$ and $16 \mathrm{~s}^{-1}$, respectively. We used syllectogram analysis to measure erythrocyte aggregation to obtain the aggregation index of the erythrocytes. A detailed description of the syllectogram can be found in the literature ${ }^{(21)}$.

\section{Analysis of oxygen-free radicals}

A technique for in vivo determination of oxygen-free radicals by the chemiluminescence assay was introduced in our laboratory. Whole blood samples $(2 \mathrm{ml})$ were obtained by transcardiac puncture with heparinised plastic syringes under pentobarbital anaesthesia in each rat after $8 \mathrm{~h}$ of fasting. Blood samples were immediately wrapped with $\mathrm{Al}$ foil to prevent light exposure and kept in an ice box until testing with the chemiluminescence assay (generally within $2 \mathrm{~h}$ ). A microplate luminometer cell activation kit (Knight Scientific Limited, Plymouth, UK) was used to measure the oxygenfree radicals in the whole blood. The microplate was placed into a luminometer (Anthos Lucy 1 Microplate Luminometer, Anthos Labtec Instrument, Wals, Austria) for analysis of chemiluminescence. Chemiluminescence data were expressed as counts per second.

\section{Statistical analysis}

Results are expressed as means and standard deviations. Data were analysed by a one-way ANOVA followed by Duncan's post hoc test to compare the experimental groups and Con with the SAS package version 8.1 (SAS Institute, Cary, NC, USA). A value of $P<0.05$ was selected as indicating statistical significance.

\section{Results}

Table 2 shows the blood biochemical and haematological variables measured at the end of the 8-week treatment with standard or high-fat diets. TC in the serum of the USFA 
Table 2. Biochemical and haematological parameters and biomass of rats fed different high-fat diets in the control group (Con) and the experimental groups (unsaturated fatty acids (USFA), SFA and trans-fatty acids (TFA)) for 8 weeks

(Mean values and standard deviations from three replicates, $n 10$ rats)

\begin{tabular}{|c|c|c|c|c|c|c|c|c|}
\hline \multirow[b]{3}{*}{ Parameters } & \multicolumn{8}{|c|}{ Diets } \\
\hline & \multicolumn{2}{|c|}{ Con } & \multicolumn{2}{|c|}{ USFA } & \multicolumn{2}{|c|}{ SFA } & \multicolumn{2}{|c|}{ TFA } \\
\hline & Mean & SD & Mean & SD & Mean & SD & Mean & SD \\
\hline Final body wt $(\mathrm{g})$ & $139 \cdot 5$ & $23 \cdot 5$ & $203 \cdot 5^{a * *}$ & 28 & $268 \cdot 9^{\mathrm{b} * *}$ & $25 \cdot 4$ & $244 \cdot 4^{\mathrm{b} * *}$ & $24 \cdot 5$ \\
\hline HDL-C (mg/l) & 503 & 113 & 417 & 34 & 503 & 109 & 470 & 32 \\
\hline LDL-C (mg/l) & 398 & 75 & 351 & 18 & 446 & 87 & 373 & 27 \\
\hline $\mathrm{TAG}(\mathrm{mg} / \mathrm{l})$ & 633 & 73 & $799^{a}$ & 186 & $1023^{\mathrm{b} * \star}$ & 197 & $1009^{b * *}$ & 196 \\
\hline $\mathrm{TC}(\mathrm{mg} / \mathrm{l})$ & 615 & 54 & $504^{*}$ & 30 & 686 & 99 & 563 & 33 \\
\hline Fib $(\mathrm{mg} / \mathrm{l})$ & 2199 & 86 & 2306 & 152 & $2341^{*}$ & 99 & 2162 & 142 \\
\hline Hct (\%) & 39.8 & $1 \cdot 8$ & 38.4 & 0.8 & $40 \cdot 1$ & 2.5 & 39.9 & $2 \cdot 2$ \\
\hline MCV (fl) & 54 & $2 \cdot 4$ & 53.1 & 1.2 & 53.9 & $2 \cdot 2$ & $54 \cdot 8$ & 1.9 \\
\hline $\mathrm{MCH}(\mathrm{pg})$ & 20 & 0.4 & $17 \cdot 7^{\star}$ & 0.5 & $18 \cdot 80 \cdot 9$ & 0.9 & $17 \cdot 9^{\star}$ & 0.8 \\
\hline $\mathrm{MCHC}(\mathrm{g} / \mathrm{l})$ & 368 & 18 & $333^{*}$ & 3 & 349 & 8 & $328^{*}$ & 6 \\
\hline Free radical levels (chemiluminescence $\times 10^{5} \mathrm{cps}$ ) & $2 \cdot 0$ & 0.6 & $2 \cdot 2^{\mathrm{a}}$ & 0.8 & $2 \cdot 7^{\mathrm{a}}$ & 0.8 & $4 \cdot 5^{\mathrm{b} * *}$ & 1.3 \\
\hline
\end{tabular}

HDL-C, HDL cholesterol; LDL-C, LDL cholesterol; TC, total cholesterol ; Fib, fibrinogen; Hct, haematocrit; MCV, mean cell volume; $\mathrm{MCH}$, mean cell Hb; MCHC, MCH concentration, $\mathrm{cps}$, counts per second.

a,b Mean values with unlike superscript letters were significantly different $(P<0.05)$.

Mean values were significantly different compared to the control group: ${ }^{\star} P<0.05,{ }^{\star *} P<0.01$.

group was significantly lower $(P<0 \cdot 05)$ than in the other three groups. HDL-C and LDL-C remained unaffected in all groups. Plasma TAG levels in the SFA and TFA groups were found to be significantly higher $(P<0.01)$ compared with the Con and USFA groups. When comparing the Con to the USFA group, TAG levels of the USFA group were significantly higher $(P<0.05)$ than the Con group. As expected, at the end of the study, the final body weight was significantly lower $(P<0.01)$ in the Con group than in the groups of rats fed the high-fat diets containing all of the fat sources. The final body weight of the SFA and TFA groups was not different from each other, but significantly higher $(P<0.05)$ than that of the USFA group. Plasma fibrinogen levels did not differ dramatically among the groups, as shown in Table 2, except that the SFA group showed slightly higher $(P<0.05)$ levels than the others. Hct and mean cell volume values remained unchanged as compared to the Con group. The values of
$\mathrm{MCH}$ and $\mathrm{MCHC}$ were significantly decreased $(P<0.05)$ in the USFA and TFA groups as compared to the Con group. The levels of oxygen-free radicals in the USFA and SFA groups appeared slightly higher than in the Con group, but without statistical significance; whereas, the TFA group showed significantly increased $(P<0.01)$ oxygen-free radical levels compared with the other groups.

Table 3 shows the values for the haemorheological parameters measured at the end of the 8-week treatment with the standard or high-fat diets. Plasma viscosity is considered to be one of the main indicators of haemorheology. Compared with the Con diet, all three high-fat diets significantly increased plasma viscosity in the rats $(P<0.01)$; but the SFA group showed a significantly lower plasma viscosity compared with the TFA and USFA groups. Whole blood viscosity, when adjusted to a standard Hct $(45 \%)$, increased significantly $(P<0.01)$ in the groups fed high-fat diets at

Table 3. Comparisons of plasma viscosity, blood viscosity, $T_{\mathrm{K}}$ values, aggregate index (Al) and deformability index (DI) in the blood of rats fed different high-fat diets in the control group (Con) and the experimental groups (unsaturated fatty acids (USFA), SFA and trans-fatty acids (TFA) for 8 weeks

(Mean values and standard deviations from three replicates, $n 10$ rats)

\begin{tabular}{|c|c|c|c|c|c|c|c|c|}
\hline \multirow[b]{3}{*}{ Parameters } & \multicolumn{8}{|c|}{ Diets } \\
\hline & \multicolumn{2}{|c|}{ Con } & \multicolumn{2}{|c|}{ USFA } & \multicolumn{2}{|c|}{ SFA } & \multicolumn{2}{|c|}{ TFA } \\
\hline & Mean & SD & Mean & SD & Mean & SD & Mean & SD \\
\hline$\eta$ Plasma $(\mathrm{mPa} \times \mathrm{s})$ & 1.25 & 0.01 & $1.38^{a * *}$ & 0.03 & $1 \cdot 30^{b * *}$ & 0.03 & $1.40^{\mathrm{a} \star \star}$ & 0.02 \\
\hline$\eta_{35}$ blood $(\mathrm{mPa} \times \mathrm{s})$ & 5.41 & 0.77 & $6 \cdot 25^{\mathrm{a}}$ & 1.42 & $7.52^{\mathrm{b} * *}$ & 0.59 & $7 \cdot 09^{b * *}$ & 0.87 \\
\hline$\eta_{300}$ blood $(\mathrm{mPa} \times \mathrm{s})$ & $3 \cdot 11$ & 0.18 & $3.77^{a \star \star}$ & 0.45 & $3.99^{b * *}$ & 0.18 & $4.52^{b * \star}$ & 0.47 \\
\hline$\eta_{600}$ blood $(\mathrm{mPa} \times \mathrm{s})$ & $2 \cdot 683$ & 0.19 & $3.39^{a * *}$ & 0.32 & $3.57^{\mathrm{b} * *}$ & 0.13 & $3 \cdot 74^{b * *}$ & 0.29 \\
\hline$T_{\text {K300 }}$ & 0.76 & 0.01 & $0.86^{a_{\star \star *}}$ & 0.02 & $0 \cdot 86^{a \star \star}$ & 0.02 & $0.99^{b * *}$ & 0.03 \\
\hline$T_{\mathrm{K} 600}$ & 0.66 & 0.01 & $0.79^{a * \star}$ & 0.02 & $0.78^{a * *}$ & 0.02 & $0.87^{b * \star}$ & 0.02 \\
\hline $\mathrm{Al}(\%)$ & $41 \cdot 85$ & $9 \cdot 0$ & $36 \cdot 8$ & $7 \cdot 6$ & $43 \cdot 71$ & 6.89 & 38.61 & $7 \cdot 1$ \\
\hline $\mathrm{DI}$ & 0.54 & 0.02 & $0.53^{a *}$ & 0.01 & $0.49^{b * *}$ & 0.02 & $0.45^{\mathrm{c} * *}$ & 0.02 \\
\hline
\end{tabular}

$\eta$ plasma, the viscosity of plasma at steady flow; $\eta_{35}$ blood, blood viscosity at shear rate of $35 \mathrm{~s}^{-1}$ and steady flow; $\eta_{300}$ blood, blood viscosity at shear rate of $300 \mathrm{~s}^{-1}$ and steady flow; $\eta_{600}$ blood, blood viscosity at shear rate of $600 \mathrm{~s}^{-1}$ and steady flow; $T_{\mathrm{K} 300}$, erythrocyte rigidity at a shear rate of $300 \mathrm{~s}^{-1} ; T_{\mathrm{K} 600}$, erythrocyte rigidity at a shear rate of $600 \mathrm{~s}^{-1}$

a,b,c Mean values with unlike superscript letters were significantly different $(P<0.05)$.

Mean values were significantly different compared to the control group: ${ }^{\star} P<0.05$, ${ }^{\star \star} P<0.01$. 
each of the three shear rates, except for the USFA group, which did not exhibit statistically significant differences at the low shear rate of $35 \mathrm{~s}^{-1}$. In addition, the blood viscosity of the USFA group at the three shear rates appeared significantly lower than that of the SFA and TFA groups. $T_{\mathrm{K}}$ values, which represent erythrocyte rigidity (indicating internal viscosity), were significantly increased in the groups fed high-fat diets at shear rates of 300 and $600 \mathrm{~s}^{-1}(P<0 \cdot 01)$ compared with the Con group. $T_{\mathrm{K}}$ values in the TFA group were significantly higher $(P<0.05)$ compared with the USFA and SFA groups.

The aggregation index values representing erythrocyte aggregation were characterised in terms of the time-dependent gyration radius of the aggregates and their fractal dimension measured by a laser diffraction method. The aggregation index values obtained from the groups fed high-fat diets did not differ significantly from the controls, suggesting the diets high in fat (whether USFA, SFA or TFA) did not have a significant effect on the development of erythrocyte aggregation. The DI values indicating erythrocyte deformability have been recognised as a determinant of microvascular perfusion. The laser diffraction method was also used to determine the DI values, which were measured by erythrocyte elongation under increasing shear stress. Table 3 shows that the DI values of the groups fed high-fat diets significantly decreased compared with the Con group. It is interesting to note that the high-fat diets decreased the DI values in rats with a rank order of TFA $>$ SFA $>$ USFA, indicating the TFA group showed the most significant reduction $(P<0 \cdot 01)$ in the DI values as compared with the other groups.

\section{Discussion}

We have found that rats fed diets high in SFA and TFA showed only minor changes in the levels of TC, HDL-C, LDL-C, Hct and mean cell volume, but a significant increase in TAG in the present study. These results are consistent with those of previous studies ${ }^{(22,23)}$ showing that rats fed high-fat diets had elevated levels of plasma TAG, while the levels of plasma TC remained unaffected ${ }^{(23)}$. The present results also agree with those of Huang et al. ${ }^{(24)}$ who found a slight (but NS) decrease in TC in rats after they had consumed a diet containing TFA for 8 weeks. Interestingly, after 16 weeks, the rats in the report by Huang et al. showed decreased TC and HDL. In contrast, the USFA group fed the diet high in soyabean oil in the present study showed a significant reduction $(P<0.05)$ in TC levels and NS difference in plasma TAG levels compared to the controls. Similar results have been found in a study of rats fed for 8 weeks with a diet that contained high levels of rapeseed oil (approximately $(40 \% \mathrm{~kJ})$, which was similar to the proportion of calories from fat in the present study) in which TC levels decreased with a slight increment in TAG and unchanged HDL-C levels compared with the low maize oil diet (approximately $(6 \% \mathrm{~kJ}))^{(25)}$.

In the present study, the fibrinogen and Hct levels in the blood showed NS differences between the Con group and the groups fed high-fat diets, which agrees with previous findings that high-fat diets do not seem to regulate the plasma level of fibrinogen ${ }^{(26)}$. The internal viscosity of the erythrocyte membrane can be determined either by MCHC or the $T_{\mathrm{K}}$, which is thought to reflect their deformability.
Curiously, we found that the $T_{\mathrm{K}}$ values of the rats fed USFA and TFA were higher, whereas the MCHC values were significantly lower than in the controls. Reports have shown that hypertriglyceridaemia cause can cause a spurious rise or fall in $\mathrm{MCH}$ or $\mathrm{MCHC}$ levels because of turbidity interference ${ }^{(27,28)}$. Moreover, limited evidence was found that high-fat diets or hypertriglyceridaemic obesity may lead to significant changes in $\mathrm{MCH}$ or MCHC values ${ }^{(29)}$.

In general, increased plasma viscosity is correlated significantly with serum fibrinogen or TAG levels ${ }^{(30)}$. However, the present results showed that increased plasma viscosity of the groups fed high-fat diets did not correlate with the (unchanged) fibrinogen levels, but did correlate with the serum TAG levels. It has been shown that the strength of the correlation between serum TAG levels and plasma viscosity was greater than that observed between LDL-C and plasma viscosity ${ }^{(31)}$. On the other hand, under steady flow conditions, shear rates of 600,300 and $35 \mathrm{~s}^{-1}$ represented high, medium and low shear rates, respectively. From the microscopic point of view, erythrocytes with impaired deformability produce elevated viscosity in the high shear rate conditions; while an increased tendency for erythrocytes to aggregate is responsible for increased blood viscosity at low shear rates. Therefore, it is plausible that the decreased erythrocyte deformation of the groups fed high-fat diets contributed to their increased blood viscosity at the high shear rate. However, the groups fed high-fat diets also showed increased blood viscosity at the low shear rate, although their erythrocyte aggregation indices were unchanged. The mechanism of erythrocyte aggregation is an extremely complicated process and depends on the interaction (attractive) force between fibrinogen and the erythrocytes, which is correlated with the surface charge distribution, surface geometry, shape and fibrinogen levels. Despite several clinical studies that have shown that high-fat meals could increase plasma TAG levels and enhance erythrocyte aggregation in human serum or plasma $^{(32,33)}$ the high-fat diets fed to the rats in the present study did not cause significant alterations in fibrinogen levels and erythrocyte aggregation. This suggests that there was NS increment in the adhesive interaction between erythrocytes and fibrinogens caused by high-fat diets fed to these rats.

Erythrocyte deformability is thought to have a vital influence on peripheral microcirculation, which depends on the passage of erythrocytes through narrow capillaries and the reduction in blood viscosity under high shear rates. The present results show that erythrocyte deformability (as shown by increased $T_{\mathrm{K}}$ values) of the groups fed high-fat diets was inversely proportional to the serum TAG levels. It had been reported that the impairment of the erythrocyte membranes found in spontaneously hypertriglyceridaemic rats may be partially responsible for the haemorheological and morphologic abnormalities that provoke a decrease in erythrocyte deformability and hyperviscosity of the blood ${ }^{(29,30)}$. Investigations on adipocyte plasma membrane fatty acid composition and fluidity showed that dietary SFA did not alter the fluidity, whereas dietary TFA resulted in decreased membrane fluidity ${ }^{(34)}$. Ibrahim et al. ${ }^{(34)}$ suggested that the decrease in membrane fluidity in the group fed trans-fats could be due to the isomeric configuration of the incorporated 
elaidic acid, which was the major TFA contained in our TFA diet (approximately $11.3 \%$ of fatty acids). In addition, it is not certain whether the trans-fat content of the membrane would be large enough to significantly alter its structure and function and lead to alterations in deformability. Niu et al. ${ }^{(35)}$ provided evidence that phospholipids derived from TFA had a higher affinity for membrane cholesterol than their cis-analogues. They also showed that membranes containing TFA phospholipids exhibited a higher acyl chain packing order and a higher transition temperature for thermal denaturation of the membrane proteins. This could probably explain why incorporation of even a small amount of $\operatorname{TFA}^{(36,37)}$ into the erythrocyte membrane may result in a significant alteration in membrane structure leading to changes in the internal viscosity and membrane deformability ${ }^{(38)}$

It is interesting to note that the TFA groups exhibited significantly higher levels of oxygen-free radicals compared with the other three groups. To avoid interference by lipid peroxidation in the analysis of oxygen-free radicals in vivo, we carefully checked the quality of dietary fats used in the high-fat diets before feeding the animals and found NS difference in oxidative stability among them. Previous studies have shown that hyperlipidaemia was correlated with the generation of free radicals ${ }^{(39)}$, possibly as a result of NADPH oxidase-associated overproduction of free radicals and inflammation ${ }^{(40)}$. The activation of phagocytes via their interaction with certain proinflammatory mediators results in the assembly of the NADPH oxidase, which catalyses the production of large amounts of the superoxide anion radical $^{(41)}$. A study of apoptosis reported that TFA could be potent inducers of apoptosis and free radical production in endothelial cells ${ }^{(42)}$. A recent study has revealed that TFA enhanced neutrophil phagocytic capacity, superoxide $\left(\mathrm{O}_{2}^{--}\right)$ and $\mathrm{H}_{2} \mathrm{O}_{2}$ production ${ }^{(43)}$. An excess of free radicals may lead to a decrease in erythrocyte deformability via protein degradation and lipid oxidation ${ }^{(16)}$. Some evidence indicates that internally generated free radicals affect erythrocyte deformability without an effect on their aggregation behavior $^{(17)}$. This is consistent with the present findings of a high level of oxygen-free radicals, impaired erythrocyte deformability and unchanged erythrocyte aggregation in the rats fed the diet high in TFA, although our data do not prove that the free radicals were generated internally in the erythrocyte membrane. Therefore, it is plausible to assume that the significant reduction in erythrocyte deformability in the rats fed the high-fat diet containing TFA could be not only the result of alterations in membrane structure due to incorporation of TFA, but also to oxidative damage produced by TFA-induced free radicals. The present haemorheological study provides new insights into the potential adverse effects of trans-fat and intake of high-fat diets on the development of CVD. Much additional research is needed to clarify the role of TFA in modulation of pro-inflammatory cytokines, their relation to erythrocyte membrane properties and the fundamental differences between TFA, SFA and USFA in membrane composition.

In summary, the present study demonstrates that rats fed high-fat diets exhibit higher serum TAG levels, plasma viscosity, whole blood viscosity and internal viscosity compared to the controls. Furthermore, rats fed a high-fat diet containing TFA showed a significant decrease in erythrocyte deformability and a higher content of oxygen-free radicals compared to the controls and rats fed diets high in other fats, suggesting the impaired erythrocyte deformability developed as a result of the attack of free radicals due to TFA-induced inflammation. These impairments could, in part, contribute to the development of CVD, which, as we know, is greatly enhanced by high-fat diets, especially those containing TFA.

\section{Acknowledgements}

The authors gratefully acknowledge the National Science Council of Executive Yuan, Taiwan, for financially supporting the present work under Grant no. NSC-97-2313-B-264-002MY2. C.-J. T. performed the animal testing and haematological analysis; C.-H. C. performed the analysis of fatty acid composition; H.-H. C. collated the data; H.-J. L. planned the paper, haemorheological analysis and writing. The authors declare that there are no conflicts of interest regarding the present paper. Ethical approval. The present research was reviewed and approved by the Institutional Animal Care and Use Committee, Yuanpei University (no. 96024).

\section{References}

1. Damjanovi M \& Barton M (2008) Fat intake and cardiovascular response. Curr Hypertens Rep 10, 25-31.

2. Dodds AJ, Boyd MJ, Allen J, et al. (1980) Changes in red cell deformability and other haemorheological variables following myocardial infarction. Br Heart J 44, 508-511.

3. Lipowsky HH (2005) Microvascular rheology and hemodynamics. Microcirculation 12, 5-15.

4. Lowe GD, Rumley A, Whincup PH, et al. (2002) Hemostatic and rheological variables and risk of cardiovascular disease. Semin Vasc Med 2, 429-439.

5. Steiner S, Jax T, Evers S, et al. (2005) Altered blood rheology in obstructive sleep apnea as a mediator of cardiovascular risk. Cardiology 104, 92-96.

6. Tabata H, Saequsa Y \& Matsuzawa T (2002) Haemorheological characterisation of a model of diet-induced hypercholesterolaemia in rats. Comp Clin Pathol 11, 160-167.

7. Sloop GD \& Garber DW (1997) The effects of low-density lipoprotein and high-density lipoprotein on blood viscosity correlate with their association with risk of atherosclerosis in humans. Clin Sci 92, 473-479.

8. Craven A, Tomaghi G, Paganardi L, et al. (1990) Weight loss does not affect the hemorheological and fibrinolytic changes in obesity. Clin Hemorheol 10, 541-546.

9. Craveri A, Tornaghi G, Paganardi L, et al. (1988) Hemorheological changes in obesity. Clin Hemorheol 8, 723-736.

10. Allison DB, Egan SK, Barraj LM, et al. (1999) Estimated intakes of trans fatty and other fatty acids in the US population. $J$ Am Diet Assoc 99, 166-174.

11. Nestel P, Noakes M, Belling B, et al. (1992) Plasma lipoprotein lipid and $\mathrm{Lp}[\mathrm{a}]$ changes with substitution of elaidic acid for oleic acid in the diet. J Lipid Res 33, 1029-1036.

12. Katan MB, Zock PL \& Mensink RP (1995) Trans fatty acids and their effects on lipoproteins in humans. Annu Rev Nutr 15, 473-493.

13. Mozaffarian D, Pischon T, Hankinson SE, et al. (2004) Dietary intake of trans fatty acids and systemic inflammation in women. Am J Clin Nutr 79, 606-612.

14. Lopez-Garcia E, Schulze MB, Meigs JB, et al. (2005) Consumption of trans fatty acids is related to plasma 
biomarkers of inflammation and endothelial dysfunction. $J$ Nutr 135, 562-566.

15. Harvey KA, Arnold T, Rasool T, et al. (2008) Trans-fatty acids induce pro-inflammatory responses and endothelial cell dysfunction. Br J Nutr 99, 723-731.

16. Srour MA, Bilto YY, Juma M, et al. (2000) Exposure of human erythrocytes to oxygen radicals causes loss of deformability, increased osmotic fragility, lipid peroxidation and protein degradation. Clin Hemorheol 23, 13-21.

17. Baskurt OK, Temiz A \& Meiselman HJ (1998) Effect of superoxide anions on red blood cell rheologic properties. Free Radic Biol Med 24, 102-110.

18. Liu DZ, Chien SC, Tseng LP, et al. (2004) The influence of hyperbaric oxygen on hemorheological parameters in diabetic rats. Biorheology 40, 605-612.

19. Dintenfass L (1975) Problems associated with definition of plasma viscosity and effect volume of red cells in blood viscosity equation. Biorheology 12, 1480-1486.

20. Schonbein HS, Ruef P \& Linderkamp O (1996) The shear stress diffractometer rheodyn SSD for determination of erythrocyte deformability: principle of operation and reproducibility. Clin Hemorheol Microcirc 16, 745-748.

21. Hardeman MR, Goedhart PT, Dobbe JGG, et al. (1994) Laserassisted optical rotational cell analyzer: a new instrument for measurement of various structural hemorheological parameters. Clin Hemorheol Microcirc 14, 605-618.

22. Lichtenstein AH (1998) Trans fatty acids and blood lipid levels, Lp(a), parameters of cholesterol metabolism, and hemostatic factors. J Nutr Biochem 9, 244-248.

23. Kempaiah RK \& Srinivasan K (2004) Influence of dietary curcumin, capsaicin and garlic on the antioxidant status of high fat fed rats. Annu Nutr Metab 48, 314-320.

24. Huang Z, Wang B, Pace R, et al. (2009) Trans fat intake lowers total cholesterol and high-density lipoprotein cholesterol levels without changing insulin sensitivity index in Wistar rats. Nutr Res 29, 206-212.

25. Ellis J, Lake A \& Hoover-Plow J (2002) Monounsaturated canola oil reduces fat deposition in growing female rats fed a high or low fat diet. Nutr Res 22, 609-621.

26. Slabber M, Barnard HC, Kuyl JM, et al. (1992) Effect of a short-term very low calorie diet on plasma lipids, fibrinogen, and factor VII in obese subjects. Clin Biochem 25, 334-335.

27. Zandecki M, Genevieve F, Gerard J, et al. (2007) Spurious counts and spurious results on haematology analysers: a review. Part I: platelets. Int J Lab Hematol 29, 4-20.

28. Savage RA (1989) Analytic inaccuracy resulting from hematology specimen characteristics: three cases of clinically misleading artifacts affecting white blood cell and platelet counts. Am J Clin Chem 92, 295-299.

29. Hernändez GN, Dabin C, del C Gayol M, et al. (2002) Haemorheological variables in a rat model of hypertriglyceridaemic obesity and diabetes. Vet Res Commun 26, 625-635.

30. Zhao T, Guo J, Li H, et al. (2006) Hemorheological abnormalities in lipoprotein lipase deficient mice with severe hypertriglyceridemia. Biochem Biophys Res Commun 341, 1066-1071.

31. Lowe GD, Wood DA, Douglas JT, et al. (1991) Relationships of plasma viscosity coagulation and fibrinolysis to coronary risk factors and angina. Thromb Haemost 65, 339-343.

32. Cicha I, Suzuki Y, Tateishi N, et al. (2001) Enhancement of red blood cell aggregation by plasma triglycerides. Clin Hemorheol Microcirc 24, 247-255.

33. Maeda N, Cicha I, Tateishi N, et al. (2006) Triglyceride in plasma: prospective effects on microcirculatory functions. Clin Hemorheol Microcirc 34, 341-346.

34. Ibrahim A, Natrajan S \& Ghafoorunissa R (2005) Dietary transfatty acids alter adipocyte plasma membrane fatty acid composition and insulin sensitivity in rats. Metabolism 54, 240-246.

35. Niu SL, Mitchell DC \& Litman BJ (2005) Trans fatty acid derived phospholipids show increased membrane cholesterol and reduced receptor activation as compared to their cis analogs. Biochemistry 44, 4458-4465.

36. Sun Q, Ma J, Campos H, et al. (2007) A prospective study of trans fatty acids in erythrocytes and risk of coronary heart disease. Circulation 115, 1858-1865.

37. Lemaitre RN, King IB, Raghunathan TE, et al. (2002) Cell membrane trans-fatty acids and the risk of primary cardiac arrest. Circulation 105, 697-701.

38. Ibrahim SA \& Ghafoorunissa SA (2001) Influence of dietary partially hydrogenated fat high in trans fatty acids on lipid composition and function of intestinal brush border membrane in rats. J Nutr Biochem 12, 116-120.

39. Henriksson P, Bergstrom K \& Edhag O (1985) Experimental atherosclerosis and a possible generation of free radicals. Thromb Res 38, 195-198.

40. Zhang X, Dong F, Ren J, et al. (2005) High dietary fat induces NADPH oxidase-associated oxidative stress and inflammation in rat. Exp Neurol 191, 318-325.

41. Elahi MM \& Matata BM (2006) Free radicals in blood: evolving concepts in the mechanism of ischemic heart disease. Arch Biochem Biophys 450, 78-88.

42. Zapolska-Downar D, Kosmider A \& Naruszewicz M (2005) Trans fatty acids induce apoptosis in human endothelial cells. J Physiol Pharmacol 56, 611-625.

43. Padovese R \& Curi R (2008) Modulation of rat neutrophil function in vitro by cis- and trans-MUFA. Br J Nutr 2, 1-9. 